\title{
High dielectric tunability, electrostriction strain and electrocaloric strength at a tricritical point of tetragonal, rhombohedral and pseudocubic phases
}

Biaolin Peng ${ }^{1,2,3}$, Qi Zhang ${ }^{4,5}$, Xing $\mathrm{Li}^{3}$, Tieyu Sun ${ }^{3}$, Shanming $\mathrm{Ke}^{1}$, Mao $\mathrm{Ye}^{1,2}$, Yu Wang ${ }^{3}$, Wei $\mathrm{Lu}^{3}$, Hanben $\mathrm{Niu}^{2}$, Xierong Zeng ${ }^{1 *}$, Huiqing Fan ${ }^{5 *}$, Haitao Huang ${ }^{3 *}$

${ }^{1}$ Shenzhen Key Laboratory of Special Functional Materials, College of Materials Science and Engineering, Shenzhen University, Shenzhen 518060, PR China

${ }^{2}$ Key Laboratory of Optoelectronic Devices and Systems of Ministry of Education and Guangdong Province, College of Optoelectronic Engineering, Shenzhen University, Shenzhen, 518060, China ${ }^{3}$ Department of Applied Physics, The Hong Kong Polytechnic University, Kowloon, Hong Kong SAR

${ }^{4}$ Department of Manufacturing and Materials, Cranfield University, Cranfield, Bedfordshire, MK43 0AL, United Kingdom

${ }^{5}$ State Key Laboratory of Advanced Technology for Materials Synthesis and Processing, Wuhan University of Technology, Wuhan 430070, Hubei, PR China

${ }^{6}$ State Key Laboratory of Solidification Processing School of Materials Science and Engineering Northwestern Polytechnical University, Xi'an 710072, China

\begin{abstract}
A relaxor ferroelectric $\mathrm{Pb}\left(\mathrm{Ni}_{1 / 3} \mathrm{Nb}_{2 / 3}\right)_{0.5} \mathrm{Zr}_{0.15} \mathrm{Ti}_{0.35} \mathrm{O}_{3}(\mathrm{PNNZT})$ ceramic was reported, designed upon an idea that the composition is selected to near a tricritical point of tetragonal, rhombohedral and pseudocubic phases. High dielectric tunability of $67 \%$,
\end{abstract}

\footnotetext{
* Author to whom correspondence should be addressed. Electronic mail: zengxier@szu.edu.cn, hqfan3@163.com, \& haitao.huang@polyu.edu.hk
} 
simultaneously with high electrostriction strain of $0.319 \%$ and high electrocaloric strength $(\Delta T / \Delta E)$ of $32.5 \mathrm{mK} \mathrm{cm} / \mathrm{kV}$ are achieved, which make it a promising multifunctional material for applications in dielectric tunable, precisely controlled and electric refrigeration devices. This is endowed by the low energy barrier among the transitions of phases and strong relaxor ferroelectric activities around the tricritical point. This work provides a guide for the design of multifunctional ferroelectric materials and can promote the development of other multifunctional ferroic materials.

Keywords: Dielectric tenability; Electrostriction; Electrocaloric; Relaxor; Morphotropic phase boundary 


\section{Introduction}

Recently, there has been an increasing interest on relaxor ferroelectric materials that possess high performance in dielectric tunability, electrostriction strain, electrocaloric effect, etc., due to the potential applications in tunable devices, electromechanical actuators, solid state cooling systems, etc[1-7]. Usually, for dielectric tunable devices, the applications demand materials owning not only high dielectric tunability but also high figure-of-merit (FOM) [8]. For electromechanical actuators, a large electrostriction strain with a high electrostriction coefficient is required[4]. For solid state cooling systems, a large electrocaloric effect with a high electrocaloric strength is also required[9].

To accomplish these requirements, most research efforts have been made. For example, to improve the FOM of titanium-containing perovskite dielectric tunable ferroelectric materials such as $(\mathrm{Ba}, \mathrm{Sr}) \mathrm{TiO}_{3}(\mathrm{~Pb}, \mathrm{Sr}) \mathrm{TiO}_{3}$, etc. $[10,11]$, due to the oxidation state of titanium being easily reduced from $\mathrm{Ti}^{4+}$ to $\mathrm{Ti}^{3+}$, strategies with doping various oxides $(\mathrm{MgO}$, etc. $)$ and ions $\left(\mathrm{Mn}^{2+}\right.$ etc. $)$ were often adopted[12]. To obtain high linear electrostrictive strain with large electrostricitve coefficient, a formula scheme "coexistence of a normal ferroelectric and a relaxor ferroelectric" was proposed, and a large electrostrictive strain $(0.1 \%)$ with an electrostrictive coefficient $Q$ of $0.02 \mathrm{~m}^{4} / \mathrm{C}^{2}$ was observed in the $(\mathrm{Sr}, \mathrm{Bi}, \mathrm{Na}) \mathrm{TiO}_{3}$ solid solution[4]. Furthermore, a large electrocaloric effect with large electrocaloric strength was expected to be realized near the ferroelectric Curie temperature, such as a large $\Delta T$ of $12 \mathrm{~K}$ with an electrocaloric strength of $24 \mathrm{mK} \mathrm{cm} / \mathrm{kV}$ was observed in the 
antiferroelectric $\mathrm{PbZr}_{0.95} \mathrm{Ti}_{0.05} \mathrm{O}_{3}$ thin film near the antiferroelectric to paraelectric phase transition temperature[5], and also a $\Delta T$ of $12.6 \mathrm{~K}$ with an electrocaloric strength of $6 \mathrm{mK} \mathrm{cm} / \mathrm{kV}$ in the relaxor ferroelectric polymer $\mathrm{P}(\mathrm{VDF}-\mathrm{TrFE})$ [7]. However, so far, to our knowledge, the above mentioned performances were rarely simultaneously reported and achieved in the same composition.

In this work, a relaxor ferroelectric $\mathrm{Pb}\left(\mathrm{Ni}_{1 / 3} \mathrm{Nb}_{2 / 3}\right)_{0.5} \mathrm{Zr}_{0.15} \mathrm{Ti}_{0.35} \mathrm{O}_{3}$ (PNNZT) ceramic was prepared by using a two-stage solid state reaction method. The composition was deliberately selected to near a tricritical point of tetragonal, rhombohedra and pseudocubic phases in the PNN-PT-PZ ternary system[13], as shown in Figure 1. It is found that high dielectric tunability $(66.4 \%)$ with a large FOM of 180 , simultaneously a high electrostriction strain of $0.319 \%$ with a large $Q$ value of $0.028 \mathrm{~m}^{4} / \mathrm{C}^{2}$ and high electrocaloric strength $(\Delta T / \Delta E)$ of $32.5 \mathrm{mK} \mathrm{cm} / \mathrm{kV}$ can be achieved. It is expected that the idea that the composition with multiphase coexisting can provide a guide for rapid positioning and design of multifunctional ferroelectric materials and will open new perspectives for exploitation of other multifunctional ferroic materials.

\section{Experimental procedure}

$\mathrm{Pb}\left(\mathrm{Ni}_{1 / 3} \mathrm{Nb}_{2 / 3}\right)_{0.5} \mathrm{Zr}_{0.15} \mathrm{Ti}_{0.35} \mathrm{O}_{3} \quad$ (PNNZT) ceramics was fabricated using high-purity $(99.9 \%)$ oxides via a columbite route. To avoid the formation of pyrochlore phases, the precursor of $\mathrm{NiNb}_{2} \mathrm{O}_{6}$ powder was synthesized from analytical grade powders of $\mathrm{NiO}$ and $\mathrm{Nb}_{2} \mathrm{O}_{5}$ by calcinating at $900{ }^{\circ} \mathrm{C}$ for $2 \mathrm{~h}$. $\mathrm{PbO}$ with excess lead of $5 \%, \mathrm{TiO}_{2}, \mathrm{ZrO}_{2}$, and $\mathrm{Nb}_{2} \mathrm{O}_{5}$ powders were mixed, milled and calcined at 
$900^{\circ} \mathrm{C}$ for $2 \mathrm{~h}$. After grinding for $2 \mathrm{~h}$ in an agate mortar, the calcined powders were then compacted into pellets with $10 \mathrm{~mm}$ in diameter and $1 \mathrm{~mm}$ in thickness under a $300 \mathrm{MPa}$ cold isostatic pressing and were sintered at $1225^{\circ} \mathrm{C}$ for $2 \mathrm{~h}$ in air, and then cooled naturally to room temperature. To reduce the loss of lead oxide during sintering, all pellets were buried in the same composition.

The crystal structures of the sintered pellets were monitored by X-ray diffraction (XRD, Rigaku $9 \mathrm{KW}$ Smartlab, Japan) on a diffractometer, using $\mathrm{Cu} \mathrm{K} \alpha$ radiation ( $\lambda$ $=1.5406 \AA$ ). The Raman scattering spectra were acquired by Raman spectrometer (Horiba HR800) with excitation source of $488 \mathrm{~nm}$ laser. The surface image was examined by scanning electron microscope (SEM, Model JEOL-6700F) operated at $20 \mathrm{kV} / \mathrm{cm}$. The microstructure was studied by transmission electron microscopy (TEM; JEOL JEM-2100F) operated at $300 \mathrm{kV} / \mathrm{cm}$. The density of all the pellets was measured by the Archimedes method. For the measurements of electrical properties, silver paste was painted on the polished pellets as the electrodes and fired at $550^{\circ} \mathrm{C}$ for 30 min. Dielectric permittivity measurements were carried out using an impedance analyzer (4294A, Agilent) with a measuring voltage $V_{\mathrm{ac}}=500 \mathrm{mV}$ at a heating rate of $3^{\circ} \mathrm{C} / \mathrm{min}$. The thermal stimulated current density was collected with a High Resistance Meter (4339B, Agilent) under the "zero-field-heating after $10 \mathrm{kV} / \mathrm{cm}$ field-cooling above the temperature of dielectric permittivity maximum" condition. The dc electric field dependence of the dielectric permittivity was measured at $10 \mathrm{kHz}$ and room temperature. The longitudinal strain-electric field $(S-E)$ and the polarization-electric field $(P-E)$ hysteresis loops were obtained by means of a 
ferroelectric tester (TF-2000, aix-ACCT) at $1 \mathrm{HZ}$. The electrostriction and the electrocaloric performances were calculated according to the $S-E$ and $P-E$ results, respectively. It should be pointed out that all measurements were performed by the same sample.

\section{Result and discussions}

Fig. 2(a) shows the XRD patterns of the PNNZT ceramics at three different polarization states of the as prepared, poled and depoled. All polarization states exhibited single perovskite phase and no traces from other impurity phases (pyrochlore phases, etc.) can be detected, indicating that the solid solution is formed. To investigate the coexistence of typical rhombohedral and tetragonal phases, the characteristic peaks between $43.9^{\circ}$ and $45.5^{\circ}$ can be well fitted into three peaks of $\mathrm{r}(002), \mathrm{t}(002)$ and $\mathrm{t}(200)$ by multi-peak fitting method, as shown in the inset of Fig. 2(a). The $\mathrm{r}(200)$ peak is corresponding to a rhombohedral symmetry[14-16], while the $t(002)$ and $t(200)$ peaks are corresponding to a tetragonal symmetry. Moreover, the tetragonal phase can be transformed to the rhombohedral phase when poled, and the induced tetragonal phase can be reverted to the rhombohedral phase when depoled, as shown by the changes of intensities and positions of the three characteristic peaks. This is the benefit of the mobility and polarization of ferroelectric active ions near the MPB with a mixed phase region rather than a critical line, where the structural energies of the coexisted ferroelectric phases are nearly equal.

In order to understand the structure change of the PNNZT relaxor ferroelectric ceramics, Raman scattering spectrum was measured from $100 \mathrm{~cm}^{-1}$ to $900 \mathrm{~cm}^{-1}$ at 10 
$\mathrm{K}$ interval in the temperature range between $293 \mathrm{~K}$ and $453 \mathrm{~K}$. Representative plots of them are shown in Fig. 2(b). Previous research work suggests that the polar $\left[\mathrm{ZrO}_{6}\right]$ and especially $\left[\mathrm{TiO}_{6}\right]$ clusters are responsible for the $\mathrm{A}_{1}\left(\mathrm{LO}_{3}\right) / \mathrm{E}\left(\mathrm{LO}_{4}\right)$ modes (around $718 \mathrm{~cm}^{-1}$ ) and the presence of dissimilar ions at the centre of the octahedral creating asymmetry is responsible for the breathing-like mode $A_{1 g}$ (around $800 \mathrm{~cm}^{-1}$ ).[17] Obviously, the $\mathrm{A}_{1}\left(\mathrm{LO}_{3}\right) / \mathrm{E}\left(\mathrm{LO}_{4}\right)$ modes are activated by the structural distortion of the lattice, while the Raman active $\mathrm{A}_{1 \mathrm{~g}}$ mode is driven by the chemical nature. It can be seen that the scattering intensity of all modes except the $\mathrm{A}_{1}\left(\mathrm{LO}_{3}\right) / \mathrm{E}\left(\mathrm{LO}_{4}\right)$ and $\mathrm{A}_{1 \mathrm{~g}}$ modes decreased sharply first and then degraded gradually with the increase of temperature, as shown by the curve of the scattering intensity versus temperature of the representative mode $\mathrm{A}_{1}\left(\mathrm{LO}_{2}\right)$ (see the inset of Fig. 2(b)) [18]. Different to that of the representative mode $A_{1}\left(\mathrm{LO}_{2}\right)$, there are three obvious peaks appeared on the curve of the scattering intensity versus temperature for the $\mathrm{A}_{1}\left(\mathrm{LO}_{3}\right) / \mathrm{E}\left(\mathrm{LO}_{4}\right)$ and $\mathrm{A}_{1 \mathrm{~g}}$ modes. Their positions are about at $333 \mathrm{~K}, 373 \mathrm{~K}$ and $413 \mathrm{~K}$, respectively. Moreover, the scattering intensity of the $\mathrm{A}_{1}\left(\mathrm{LO}_{3}\right) / \mathrm{E}\left(\mathrm{LO}_{4}\right)$ modes is stronger than that of the $\mathrm{A}_{1 \mathrm{~g}}$ modes in the whole measuring temperature range, indicating that the contribution from the structural distortion of the lattice is much larger than that from the chemical nature.

Fig. 2(c) shows the SEM surface image of the PNNZT ceramics sintered at 1225 ${ }^{\circ} \mathrm{C}$. The sample exhibits a well crystallized and dense microstructure, as well as a wide grain size of 1.5 to $5 \mu \mathrm{m}$. Meanwhile, terraced stripes can be observed on its surface of each grain, indicating that a step growth mode is employed. The EDS 
results show that the composition of the sample is consistent with its chemical formula, as shown in the inset of Fig. 2(c).

To further get an insight into the microstructure of the PNNZT ceramics, TEM analysis was carried out, as shown in Fig. 2(d). Tetragonal twin-like $90^{\circ}$ domains with light or dark contrast can be readily seen, as marked by arrow 2 and 3[19]. A complex herringbone domain structure derived from the coexistence of tetragonal and rhombohedral phases can also be observed, as signed by arrow 1[19]. Furthermore, a small amount of nanodomains originated from the coexistence of pseudocubic and rhombohedral can also be detected near the junction of triple grain boundary, as shown by arrow 4[19]. Based on the above analyses and combined with the analysis result of XRD, it can be believed that the composition of the designed PNNZT ceramic is in a region of three phases coexisted. Therefore, it should be pointed that the phase transition induced by the electric field in Fig. 2(a) for the PNNZT ceramic not only originated from the contribution of tetragonal phase to rhombohedral phase, but also some of them maybe come from the contribution of pseudocubic phase to rhombohedral phase. In a word, it is very complex phase transition process.

Fig. 3 shows the dependence of the dielectric permittivity $(\varepsilon(T))$, the dielectric losses $(\tan \delta(T))$ and the thermal stimulated current $J(T)$ vs. temperature of PNNZT ceramic. The sample exhibited a typical relaxor behavior, namely the temperature $\left(T_{\mathrm{m}}\right)$ of dielectric permittivity maximum as well as the temperature $\left(T_{\mathrm{m}}{ }^{\delta},\right)$ of maximum dielectric losses are shifted towards higher temperatures with increasing frequency. Meanwhile, dielectric dispersion appears in the vicinity and below $T_{\mathrm{m}}$, whereas the 
maximum values of dielectric losses increase with increasing frequency[20-22]. The relaxor behavior is considered to be closely related to the response of polar nanoregions (PNRs) which are characterized by the nano-sized polar islands with multiple homogeneities embedded in an average cubic matrix[23]. Furthermore, on the curve of the $J(T)$, a thermal depolarization temperature $\left(T_{\mathrm{d}}\right)$ with a current maximum of $0.35 \mathrm{~mA} / \mathrm{m}^{2}$ was observed at $398 \mathrm{~K}$, which is slightly higher than the temperature of the dielectric losses maximum. It has been well documented that the gap between $T_{\mathrm{m}}$ and $T_{\mathrm{d}}\left(T_{\mathrm{m}}-T_{\mathrm{d}}\right)$ reflects the degree of the electric dipole order in relaxor ferroelectric ceramics[24]. The smaller $T_{\mathrm{m}}-T_{\mathrm{d}}$ is, the greater the degree of the electric dipole order is. The value of the $T_{\mathrm{m}}-T_{\mathrm{d}}$ at $10 \mathrm{kHz}$ is only $10 \mathrm{~K}$, indicating that a large electric dipole order or strong ferroelectric activities is accompanied with the PNNZT ceramic.

Fig. 4 shows the dependence of the $\varepsilon$ and $\tan \delta$ vs. dc bias electric field at room temperature and $10 \mathrm{kHz}$. It can be seen that both the $\varepsilon$ and the $\tan \delta$ decrease sharply with the increase of the bias dc electric field, indicating that there is a high dielectric non-linear electric field dependence. Dielectric tunability $(\eta)$ and Figure-of-merit $(F O M)$ can be defined as follows: $(\varepsilon(0)-\varepsilon(E)) / \varepsilon(0)$, and tunability $/ \tan \delta$, respectively[25]. They both increase with increasing electric field, as shown in the inset of Fig. 4. A high tunability of $67 \%$ and a large FOM of 180 are achieved at $E=$ $15.9 \mathrm{kV} / \mathrm{cm}$, which are comparable to those (tunability of $12 \%$ and FOM of 200 at $E$ $=30 \mathrm{kV} / \mathrm{cm}$ ) of the $\mathrm{Ba}_{0.4} \mathrm{Sr}_{0.6} \mathrm{TiO}_{3}$ ceramics[26], those of (tunability of $45 \%$ and FOM of 225 at $E=40 \mathrm{kV} / \mathrm{cm}$ ) of the $\mathrm{BaZr}_{0.3} \mathrm{Ti}_{0.7} \mathrm{O}_{3}$ ceramic[27], indicating that the PNNZT 
ceramic is a very promising for the application in dielectric tunable devices. Furthermore, the $\varepsilon(E)$ of the PNNZT relaxor ferroelectric ceramic can be described by the following equation[28].

$\varepsilon(E)=\varepsilon_{r}(0) /\left\{1+\lambda\left[\varepsilon_{0} \varepsilon_{r}(0)^{3} E^{2}\right]\right\}^{1 / 3}+\left(P_{r} x / \varepsilon_{0}\right)[\cosh (E x)]^{-2}, x=P_{r} L^{3} /\left(2 k_{B} T\right)$

where $\varepsilon_{0}$ is the dielectric permittivity of free space, $\lambda$ a temperature-independent coefficient, $\varepsilon_{r}(0)$ the relative dielectric permittivity at zero field, $P_{r}$ the polarization of a polar nanocluster, $L$ the cluster size (or correlation length), $k_{B}$ the Boltzmann's constant. The first term in Eq. (1) represents the polarization contribution from lattice phonons (Johnson contribution), and the second stands for the re-orientation contribution from polar nanoregions (Langevin contribution). The black solid line fitted according to Eq. (1) agrees quite well with the experimental data. Obviously, the nanoclusters make a significant contribution to $\varepsilon$ below $\sim 6 \mathrm{kV} / \mathrm{cm}$, which decreases quickly with increasing $E$ and almost disappears at an electric field above $9 \mathrm{kV} / \mathrm{cm}$. It can also be seen that the dielectric permittivity contributed by the nanoclusters $\left(\varepsilon_{c l u s t e r}\right)$ is 1144 under zero bias, about $20.06 \%$ of the total dielectric permittivity $\left(\varepsilon_{\text {total }}\right)$. The size of polar nanoregions, according to the fitting, is $L=12.6 \mathrm{~nm}$.

Fig. 5 shows the electric field dependence of the strain $\left(S_{\text {strain }}(E)\right)$ of PNNZT ceramic at selected temperatures. A high strain level of $0.319 \%$ is obtained at room temperature and $40 \mathrm{kV} / \mathrm{cm}$. This strain level is comparable with those of other lead-containing system[29-32]. For the linearity between $S_{\text {strain }}$ and $E$, the piezoelectric constants $\left(\mathrm{d}_{33} *\right)$ is calculated as $1197 \mathrm{pm} / \mathrm{V}$ from the slope of the origin of the $S_{\text {strain }} E$ curve, as shown by the red solid line in Fig. 5. Also, the high piezoelectric activities 
$\left(d_{33}\right)$ of the sample can be measured after dc electric field poling, and the obtained $d_{33}$ value is about $608 \mathrm{pC} / \mathrm{N}$, as shown in the inset (a) of Fig. 5. With the increase of the temperature, the $S_{\text {strain }}(E)$ become more and more hysteresis free, indicating an increase of the relaxor contribution from the polar nanoregions[33].

It is known that the electrostricitve strain follows the theoretically derived quadratic relation[30, 34, 35]:

$S_{\text {strain }}=Q P^{2}$

where $Q$ is the electrostriction coefficient. The strain versus polarization squared $\left(P^{2}\right)$ is plotted in the inset (b) of Fig. 5. It can be seen that the profile of $S$ versus $P^{2}$ can be fitted well to a straight line in the whole electric-field range, as well as the measured temperature range. This indicates that the electrostriction coefficient does not depend on the strength of electric field and on temperature. From the fitting of the above branch of the $S-P^{2}$ at $308 \mathrm{~K}$ shown by the red solid line in the inset (b) of Fig. 5, a large $Q$ value of $0.028 \mathrm{~m}^{4} / \mathrm{C}^{2}$ is obtained for the sample, which is also comparable with those of other lead-containing system[29-32]. Furthermore, it can be observed that the slope of the $S-P^{2}$ remains almost unchanged with the increase of the temperature, indicating that a high thermal stability of the $Q$ is obtained. The excellent electrostriction performance of the PNNZT ceramic may make it an attractive material for application in precisely controlled devices.

In order to evaluate the electrocaloric effect (ECE) in the PNNZT ceramic, the electric field dependence of the polarization $(P-E)$ loops at $1 \mathrm{~Hz}$ were measured at a $10 \mathrm{~K}$ interval in the temperature range between $308 \mathrm{~K}$ and $447 \mathrm{~K}$. Representative 
plots of $P-E$ loops are shown in Fig. 6. Obviously, the shape of $P-E$ loop becomes more and more slender with the increase of temperature. The temperature dependence of the polarization $(P(T))$ at selected electric field values is extracted from the upper branches of the $P-E$ hysteresis loops in $E>0$ and presented in the inset (a) of Fig. 6. The solid lines in the inset (a) of Fig. 6 represent cubic-spline interpolations of the raw experimental data. In order to estimate the ECE of the PNNZT ceramic, the curves of pyroelectric coefficients versus temperature $\left((\partial P / \partial T)_{E}(T)\right)$ at selected electric field values were derived from the differential values of $P(T)$ curves and plotted in the inset (b) of Fig. 6. Assuming that the Maxwell relationship $(\partial P / \partial T)_{E}=$ $\left(\partial S_{\text {entropy }} / \partial E\right)_{T}$ is established, reversible adiabatic changes in temperature $(\Delta T)$ and entropy $\left(\Delta S_{\text {entropy }}\right)$ for a material of density $\rho$ with heat capacity $C$ are estimated by follows $[5,7]$ :

$$
\begin{gathered}
\Delta T=-\frac{1}{\rho} \int_{E_{1}}^{E_{2}} \frac{T}{C}\left(\frac{\partial P}{\partial T}\right)_{E} d E \\
\Delta S_{\text {entropy }}=-\frac{1}{\rho} \int_{E_{1}}^{E_{2}}\left(\frac{\partial P}{\partial T}\right)_{E} d E
\end{gathered}
$$

Where $T$ is the operating temperature, $P$ is the maximum polarization at the applied electric field $E$, and $E_{1}$ and $E_{2}$ are the initial and final applied electric field, respectively. In the temperature range of interest for the PNNZT ceramics the heat capacity $C$ and the density $\rho$ are $0.3 \mathrm{~J} \cdot(\mathrm{K} \cdot \mathrm{g})$ and $7.9 \mathrm{~g} / \mathrm{cm}^{3}$, respectively. The adiabatic temperature change $\Delta T$ and entropy change $\Delta S_{\text {entropy }}$ of the PNNZT ceramic under different electric field changes $\Delta E$ are shown in Fig. 7 and its inset (a), respectively. Peak $\Delta T=1.3 \mathrm{~K}$ at $40 \mathrm{kV} / \mathrm{cm}$ was obtained at $430 \mathrm{~K}$ and near the right 
of the temperature of the dielectric maximum, as well as peak $\Delta S_{\text {entropy }}=0.87 \mathrm{~J} /(\mathrm{K} \cdot \mathrm{kg})$. Meanwhile, it can be found that the position of the $\Delta T$ or $\Delta S_{\text {entropy }}$ peak shifted to higher temperature with the increase of the applied electric field, in agreement with previous experimental and theoretical reports[36, 37], indicating that the zone of ferroelectric phases was extended under the induction of the applied electric field. Furthermore, it can be found that a small peak around $325 \mathrm{~K}$ can be observed on both $\Delta T$ and $\Delta S_{\text {entropy. }}$ The appearance of the peak near room temperature may be related to the phase transition of tetragonal or pseudocubic phase to rhombohedral phase induced by the electric field, as discussed in the part of microstructure of PNNZT ceramic.

In spite of the small value of peak $\Delta T$, however, the electrocaloric strength $(\Delta T / \Delta E=32.5 \mathrm{mK} \mathrm{cm} / \mathrm{kV})$ of the PNNZT ceramic designed to near a tricritical point of three-phase coexistence is large, as shown by the inset (b) of Fig. 7. It is comparable to that $(5 \mathrm{mK} \mathrm{cm} / \mathrm{kV})$ of the antiferroelectric $\mathrm{PbZr}_{0.95} \mathrm{Ti}_{0.05} \mathrm{O}_{3}$ ceramics, that $(6 \mathrm{mK} \mathrm{cm} / \mathrm{kV})$ of the ferroelectric polymer $\mathrm{P}(\mathrm{VDF}-\mathrm{TrFE})$ and even that $(25 \mathrm{mK}$ $\mathrm{cm} / \mathrm{kV}$ ) of the ferroelectric single crystal $0.9 \mathrm{~Pb}\left(\mathrm{Mg}_{1 / 3} \mathrm{Nb}_{2 / 3}\right) \mathrm{O}_{3}-0.1 \mathrm{PbTiO}_{3}[7,38,39]$, etc.

Moreover, by using thin-film geometry, an intrinsic ECE with bigger $\Delta T$ in PNNZT could be obtained due to a significant improve in its dielectric breakdown strength. Similar to the $E C E$, an elastocaloric change in temperature $\Delta T_{\sigma}$ for a material with heat capacity $C_{\sigma}$ is determined by[40]:

$$
\Delta T_{\sigma}=-\int_{0}^{\sigma} \frac{T}{C_{\sigma}}\left(\frac{\partial S_{\text {strain }}}{\partial T}\right)_{\sigma, E} d \sigma
$$


where the Maxwell relation $\left(\partial S_{\text {strain }} / \partial T\right)_{\sigma, E}=\left(\partial S_{\text {entropy }} / \partial \sigma\right)_{E, T}$ is assumed. For a thin-film structure, the field $\sigma$ stress can be regulated and controlled by using substrates with different lattice parameters. Based on the large diffused peak of the $\left(\partial S_{\text {strain }} / \partial T\right)_{E}(T)$, which is extracted from the polynomial fitting curve of the strain versus temperature $\left(S_{\text {strain }}(T)_{E}\right)$ of PNNZT, as shown by the red arrow in the inset (b) of Fig. 7, it is believed that a larger $E C E$ with a wide operating temperature can be achieved by coupling the elastocaloric effect[41]. Furthermore, in practical application, the peak of $\Delta T$ can be shifted to room temperature by elements doping such as $\mathrm{La}, \mathrm{Ba}$, etc[42].

\section{Conclusions}

A high dielectric tunability of $67 \%$ with a large $F O M$ of 180 , simultaneously a high electrostriction strain of $0.319 \%$ with a large $Q$ value of $0.028 \mathrm{~m}^{4} / \mathrm{C}^{2}$ and a high electrocaloric strength $(\Delta T / \Delta E)$ of $32.5 \mathrm{mK} \mathrm{cm} / \mathrm{kV}$ are observed in the tricritical point relaxor ferroelectric PNNZT ceramic with the coexistence of tetragonal, rhombohedral and pseudocubic phases. This is accomplished by the low energy barrier among the transitions of phases and strong relaxor ferroelectric activities around the tricritical point. The high dielectric tunability, electrostriction strain and electrocaloric strength of the relaxor ceramic may make it an attractive multifunctional material for applications in dielectric tunable, precisely controlled and electric refrigeration devices. The exploration of tricritical point in multi-component phase diagram maybe can provide a guide for the design of multifunctional ferroelectric materials, as well as other multifunctional ferroic materials. 


\section{Acknowledgements}

This work was supported by the Hong Kong Polytechnic University (Project Nos.:

A-PL54, G-UC69 and 1-ZVCG), the National Natural Science Foundation of China (51402196, 51272161, 51172187, 51202150), the China Postdoctoral Science Foundation (2014M552229), and the Shenzhen Science and Technology Research Foundation (CXB201005240010A), 111 Program (B08040) of MOE,.

\section{References}

[1] L. Song, Y. Chen, G. Wang, L. Yang, T. Li, F. Gao, X. Dong, Ceram. Int., 40 (2014) 12573-12577.

[2] S.-X. Wang, J.-H. Hao, Z.-P. Wu, D.-Y. Wang, Y. Zhuo, X.-Z. Zhao, Appl. Phys. Lett., 91 (2007).

[3] Q. Li, G. Zhang, X. Zhang, S. Jiang, Y. Zeng, Q. Wang, Adv. Mater., 27 (2015) 2267.

[4] C. Ang, Z. Yu, Adv. Mater., 18 (2006) 103-106.

[5] A.S. Mischenko, Q. Zhang, J.F. Scott, R.W. Whatmore, N.D. Mathur, Science, 311 (2006) 1270-1271.

[6] B. Peng, H. Fan, Q. Zhang, Adv. Funct. Mater., 23 (2013) 2987-2992.

[7] B. Neese, B. Chu, S.-G. Lu, Y. Wang, E. Furman, Q.M. Zhang, Science, 321 (2008) 821-823.

[8] B. Peng, H. Fan, Q. Zhang, J. Am. Ceram. Soc., 96 (2013) 1852-1856.

[9] Y. Bai, G. Zheng, S. Shi, Appl. Phys. Lett., 96 (2010).

[10] G. Hu, F. Gao, L. Liu, B. Xu, Z. Liu, J. Alloys Compd., 518 (2012) 44-50. 
[11] L. Yang, G. Wang, X. Dong, D. Remiens, J. Am. Ceram. Soc., 93 (2010) $1215-1217$.

[12] Y. Somiya, A.S. Bhalla, L.E. Cross, Integrated Ferroelectrics, 131 (2011) 237-245.

[13] X.H. Zhu, Z.Y. Meng, J Mater Sci, 31 (1996) 2171-2175.

[14] X.P. Jiang, J.W. Fang, H.R. Zeng, G.R. Li, D.R. Chen, Q.R. Yin, J. Mater. Res., 15 (2000) 2745-2749.

[15] M.S. Yoon, H.M. Jang, S. Kim, Jpn. J. Appl. Phys., 34 (1995) 1916-1921.

[16] M.S. Yoon, H.M. Jang, J. Appl. Phys., 77 (1995) 3991-4001.

[17] V.S. Puli, A. Kumar, D.B. Chrisey, M. Tomozawa, J.F. Scott, R.S. Katiyar, J. Phys. D: Appl. Phys., 44 (2011) 395403.

[18] M. Deluca, T. Sakashita, W. Zhu, H. Chazono, G. Pezzotti, J. Appl. Phys., 101 (2007) 083526.

[19] Z. Xinhua, Z. Jianming, Z. Shunhua, L. Qi, M. Zhongyan, L. Zhiguo, M. Naiben, J. Eur. Ceram. Soc., 20 (2000) 1251-1255.

[20] A.A. Bokov, Z.G. Ye, Appl. Phys. Lett., 77 (2000) 1888-1890.

[21] A.A. Bokov, Z.G. Ye, J Mater Sci, 41 (2006) 31-52.

[22] A.A. Bokov, Y. Zuo-Guang, Journal of Advanced Dielectrics, 2 (2012) 1241010.

[23] F. Desheng, H. Taniguchi, M. Itoh, S.Y. Koshihara, N. Yamamoto, S. Mori, Phys. Rev. Lett., 103 (2009) 207601 - 207604.

[24] Z.-G. Ye, Key Eng. Mater., 155-156 (1998) 81-122.

[25] T.M. Correia, Q. Zhang, J. Appl. Phys., 108 (2010) 044107. 
[26] J. Zhang, J. Zhai, X. Yao, Scr. Mater., 61 (2009) 764-767.

[27] X.G. Tang, K.H. Chew, H.L.W. Chan, Acta Mater., 52 (2004) 5177-5183.

[28] C. Ang, Z. Yu, Physical Review B, 69 (2004) 174109.

[29] P. Kumar, C. Prakash, T.C. Goel, Sci. Technol. Adv. Mater., 8 (2007) 463-468.

[30] S.E. Park, T.R. Shrout, P. Bridenbaugh, J. Rottenberg, G.M. Loiacono, Ferroelectrics, 207 (1998) 519-526.

[31] Z. Qiming, P. Wuyi, A. Bhalla, L.E. Cross, J. Am. Ceram. Soc., 72 (1989) 599-604.

[32] S.E. Park, V. Vedula, M.J. Pan, W.S. Hackenberger, P. Pertsch, T.R. Shrout, Proceedings of the SPIE - The International Society for Optical Engineering, 3324 (1998) 136-144.

[33] R. Pirc, R. Blinc, V.S. Vikhnin, Physical Review B, 69 (2004) 212105.

[34] K. Uchino, S. Nomura, L.E. Cross, S.J. Jang, R.E. Newnham, J. Appl. Phys., 51 (1980) 1142-1145.

[35] S.E. Park, T.R. Shrout, P. Bridenbaugh, J. Rottenberg, G.M. Loiacono, Ferroelectrics, 207 (1998) 519-526.

[36] Y. Bai, G.-P. Zheng, K. Ding, L. Qiao, S.-Q. Shi, D. Guo, J. Appl. Phys., 110 (2011) 094103.

[37] M.C. Rose, R.E. Cohen, Phys. Rev. Lett., 109 (2012) 187604.

[38] J. Wang, T. Yang, K. Wei, X. Yao, Appl. Phys. Lett., 102 (2013) 152907.

[39] L. Luo, H. Chen, Y. Zhu, W. Li, H. Luo, Y. Zhang, J. Alloys Compd., 509 (2011) $8149-8152$. 
[40] Y. Liu, I.C. Infante, X. Lou, L. Bellaiche, J.F. Scott, B. Dkhil, Adv. Mater., 26 (2014) 6132.

[41] M. Liu, J. Wang, Scientific reports, 5 (2015) 7728.

[42] X. Hao, J. Zhai, L.B. Kong, Z. Xu, Prog. Mater Sci., 63 (2014) 1-57. 


\section{Figure captions}

Figure 1. The selected composition used in this work and its position (the red dot) in the phase diagram of the PNN-PT-PZ ternary solid solution[13]. $\mathrm{F}_{\mathrm{T}}$ : ferroelectric tetragonal, $\mathrm{F}_{\mathrm{R}}$ : ferroelectric rhombohedral, Fpc: pseudocubic, P: paraelectric.

Figure 2. (a) XRD pattern of the PNNZT ceramics. Inset: the fitted patterns between $43.9^{\circ}$ and $45.5^{\circ}$. (b) Raman spectra of the PNNZT ceramic as a function of wave number at selected temperatures. Inset: the scattering intensities versus temperature for the selected $\mathrm{A}_{1}\left(\mathrm{LO}_{3}\right) / \mathrm{E}\left(\mathrm{LO}_{4}\right), \mathrm{A}_{1 \mathrm{~g}}$ and $\mathrm{A}_{1}\left(\mathrm{LO}_{2}\right)$ modes. (c) SEM surface image and EDS (inset). (d) TEM image. Arrow 2 and 3: tetragonal twin-like $90^{\circ}$ domains, arrow 1: herringbone domains with coexistence of tetragonal and rhombohedral, the arrow 4: nanodomains with coexistence of pseudocubic and rhombohedral.

Figure 3. $\varepsilon(T), \tan \delta(T)$ and $J(T)$ of PNNZT ceramic. $J$ is collected under the "zero-field-heating after $10 \mathrm{kV} / \mathrm{cm}$ field-cooling above the temperature of dielectric permittivity maximum" condition.

Figure 4. $\varepsilon(E)$ and $\tan \delta(E)$ of PNNZT ceramic at room temperature and $10 \mathrm{kHz}$. Symbols: experimental data, the blue short dashed dot line: the Johnson contribution from polar nanoclusters, the red short dashed line: the Langenvin contribution from lattice phonons. Inset: $\eta(E)$ and $F O M(E)$.

Figure 5. $S_{\text {strain }}(E)$ of PNNZT ceramic at selected temperatures. Inset: (a) $d_{33}$ at room temperature and (b) $S_{\text {strain }}\left(P^{2}\right)$ at selected temperatures.

Figure 6. $P-E$ loops of PNNZT ceramic at selected temperatures. Inset: (a) $P(T)$ at selected electric field values and (b) $(\partial P / \partial T)_{E}(T)$ at selected electric field values. 
Figure 7. $\Delta T(T)$ of PNNZT ceramic at selected electric fields. Inset: (a) $\Delta S_{\text {entropy }}(T)$ at selected electric fields. (b) $C O P(T)$ and $\left(\partial S_{\text {strain }} / \partial T\right)_{E}(T)$ at $40 \mathrm{kV} / \mathrm{cm}$. 
2015-06-16

\title{
High dielectric tunability, electrostriction
} strain and electrocaloric strength at a tricritical point of tetragonal, rhombohedral and pseudocubic phases

\author{
Peng, Biaolin
}

Elsevier

Zeng $X$ et al. High dielectric tunability, electrostriction strain and electrocaloric strength at a tricritical point of tetragonal, rhombohedral and pseudocubic phases, Journal of Alloys and pÿCompounds, Volume 646, 15 October 2015, Pages 597602.

https://dspace.lib.cranfield.ac.uk/handle/1826/10654

Downloaded from Cranfield Library Services E-Repository 